\title{
The High-Voltage Monolithic Active Pixel Sensor for the Mu3e Experiment
}

\section{Shruti SHRESTHA ${ }^{* \dagger}$}

Physikalisches Institut Heidelberg

E-mail: shruti@physi.uni-heidelberg.de

The Mu3e experiment searches for the lepton flavor violating decay $\mu^{+} \rightarrow e^{+} e^{-} e^{+}$with the goal of a branching fraction sensitivity below $10^{-16}$. To measure the momentum and vertex position of low momentum electrons $(10-53 \mathrm{MeV} / \mathrm{c})$ originating from this rare decay with high precision, a tracking detector built from High-Voltage Monolithic Active Pixel Sensors (HV-MAPS) is developed.

The MUPIX chip implements a HV-MAPS architecture and is produced in $180 \mathrm{~nm}$ HV-CMOS technology. HV-MAPS is the technology of choice because the chip can be thinned to $50 \mu \mathrm{m}$, is radiation-tolerant, has a high time resolution, and is low cost. Furthermore, to reduce the material budget, the pixel readout electronics are embedded inside this sensor chip which are supported by a low mass mechanical structure built from a $25 \mu \mathrm{m}$ Kapton foil.

Technology and Instrumentation in Particle Physics 2014,

2-6 June, 2014

Amsterdam, the Netherlands

\footnotetext{
${ }^{*}$ Speaker.

${ }^{\dagger}$ For the Mu3e collaboration
} 


\section{Introduction}

In the standard model (SM) lepton flavor is a conserved quantity. In the neutrino sector, lepton flavor violation (LFV) has been observed in the form of neutrino mixing [1], 2, 3]. Consequently, lepton flavor is a broken symmetry, the SM has to be adapted to incorporate massive neutrinos and hence LFV is also expected in the charged lepton sector. However, as the exact mechanism and size of charged LFV is unknown, its study is of great interest because it is associated with neutrino mass generation, CP violation and new physics beyond the SM. The LFV muon decay $\mu^{+} \rightarrow e^{+} e^{-} e^{+}$can occur via neutrino mixing in a loop as shown in figure 1. The branching fraction of this process is directly proportional to the factor $\frac{\Delta m^{2}}{M_{W}^{2}}$, where $\Delta m^{2}$ is the neutrino mass squared difference and $M_{W}$ is the $\mathrm{W}$ boson mass. Due to the very small value of $\Delta m^{2}$ compared with the electroweak mass scale, the branching fraction is suppressed to an unobservable level of $O\left(10^{-54}\right)$ in the SM. Many theories beyond the SM lead to strongly enhanced LFV branching ratios, examples are supersymmetric particles in a loop as shown in figure 2 and heavy particles which can directly couple to both the electron and the muon at tree level as in models with extended Higgs sector [丹], as shown in figure 3 .

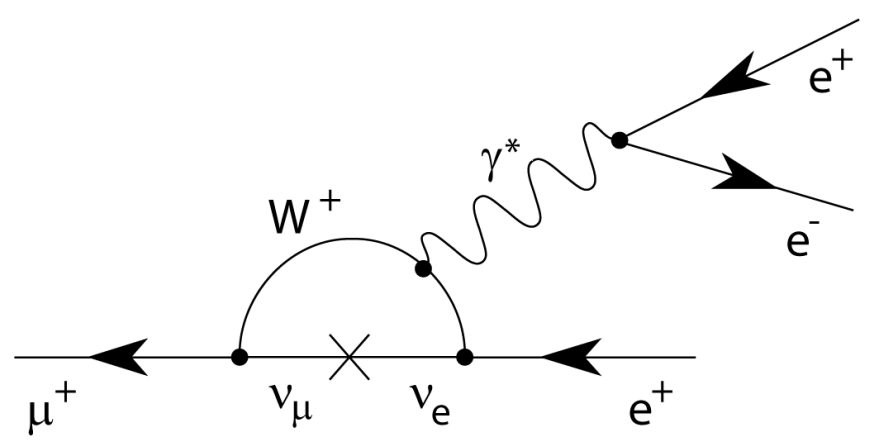

Figure 1: $\mu^{+} \rightarrow e^{+} e^{-} e^{+}$decay via neutrino mixing.

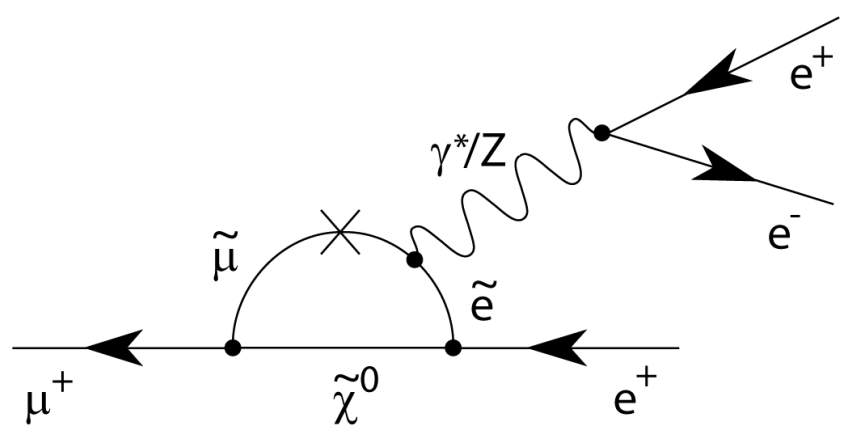

Figure 2: Lepton flavor violation $\mu^{+} \rightarrow e^{+} e^{-} e^{+}$decay involving supersymmetric particles.

The search for $\mu^{+} \rightarrow e^{+} e^{-} e^{+}$is complementary to other LFV searches i.e. indirect searches in the decay of muons such as the $\mu^{+} \rightarrow e^{+} \gamma$ [5], the conversion of a muon to an electron in the field of a nucleus [6], and direct searches performed at the Large Hadron Collider for LFV decays of a 


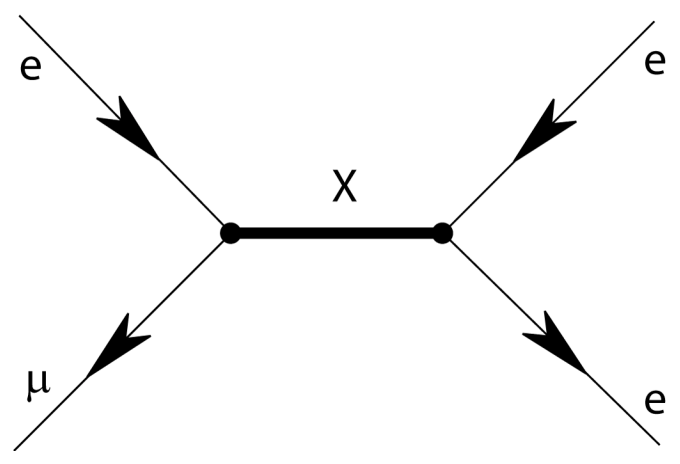

Figure 3: Lepton flavor violation at tree level.

Higgs boson to a muon-tau pair [7]. The Mu3e experiment is designed to search for $\mu^{+} \rightarrow e^{+} e^{-} e^{+}$ with a branching ratio sensitivity of $<10^{-16}$ which is an improvement by four orders of magnitude over the current limit [8]. This measurement requires a continuous beam intensity of more than $10^{9}$ muons per second. This proceedings outlines the challenges for the detector technology and reports the results from test beam measurements of the MUPIX chip at DESY (Hamburg, Germany) using an electron beam.

\section{Signal and background processes}

The $\mu^{+} \rightarrow e^{+} e^{-} e^{+}$signal events are constitued by two positrons and an electron with opposite curvature in a solenoidal magnetic field. The decay products should be coincident in time, originate from the same vertex and the topology should be co-planar. Since the decay products stem from a stopped muon which decays at rest, the vectorial sum of the momenta must vanish and the total invariant mass must sum to the muon mass. Furthermore, the individual energies should be less than $53 \mathrm{MeV}$.

There are two main sources of background events: the radiative muon decay with internal conversion and the combinatorial background. The latter is from Michel decay positrons $\left(\mu^{+} \rightarrow e^{+} \bar{v}_{\mu} v_{e}\right)$ together with an electron from Bhabha scattering, photon conversion or mis-reconstructed backcurl tracks. This combinatorial background can be suppressed with an excellent vertex, timing and momentum resolution. The radiative muon decay with internal conversion $\mu^{+} \rightarrow e^{+} e^{-} e^{+} \bar{v}_{\mu} v_{e}$ can only be identified through the missing energy carried away by the neutrinos. Thus a precise measurement of the momentum of the decay products is required. Hence, in order to identify background from radiative muon decays with internal conversion, a momentum resolution $<0.5 \mathrm{MeV} / \mathrm{c}$ is needed while, to minimize the combinatorial background, a precise vertex resolution of $200 \mu \mathrm{m}$ and time resolution of $\leq 100$ ps are required. Both the vertex and momentum resolution criteria lead to the requirement of extremely low material budget in the active volume to minimize multiple Coulomb scattering. Hence, the tracking detector has to be very thin with a layer thickness below $0.1 \%$ of a radiation length. This can be achieved by using High-Voltage Monolithic Active Pixel Sensors (HV-MAPS) since they can be thinned below $50 \mu \mathrm{m}$. 


\section{Muon beam}

For the Mu3e experiment, the muon beam must be of high intensity. Currently, a beam intensity about $10^{8}$ muons/s is available at the Paul Scherrer Institute in Switzerland. This beam is produced from surface muons in a carbon target using the high intensity $2.2 \mathrm{~mA}$ proton beam with $590 \mathrm{MeV} / \mathrm{c}$. This beam will be used in the phase I of the Mu3e experiment. To reach sensitivity goal of $10^{-16}$ in the branching fraction, a new beam line has been proposed. This high intensity beam line (HiMB) would make use of surface muons created in the target of the Swiss Spallation Neutron Source (SINQ) and could provide muon decay rates $>2 \times 10^{9}$ muons/s for phase II.

\section{Detector design for the Mu3e experiment}

Mu3e is a fixed target experiment based on a long tube design. The tracking detector consists of a $36 \mathrm{~cm}$ long central pixel detector around a hollow double-cone aluminum target. It is extended to both directions by two silicon pixel stations to measure the recurling decay products as shown in figure 4 . The multiple scattering effects on the measurement of momentum cancel to first order if a particle track is measured close to a semicircle [9]. The entire detector is surrounded by a $1 \mathrm{~T}$ solenoid magnet. The pixel detector allows for good vertex reconstruction and excellent momentum resolution of $\operatorname{RMS}(\mathrm{p})=0.28 \mathrm{MeV} / \mathrm{c}$ as estimated from simulation [10]. The fibre tracker and the tile detector provide time resolution in the order of $1 \mathrm{~ns}$ and $100 \mathrm{ps}$, respectively, which are suitable for the suppression of the combinatorial background.

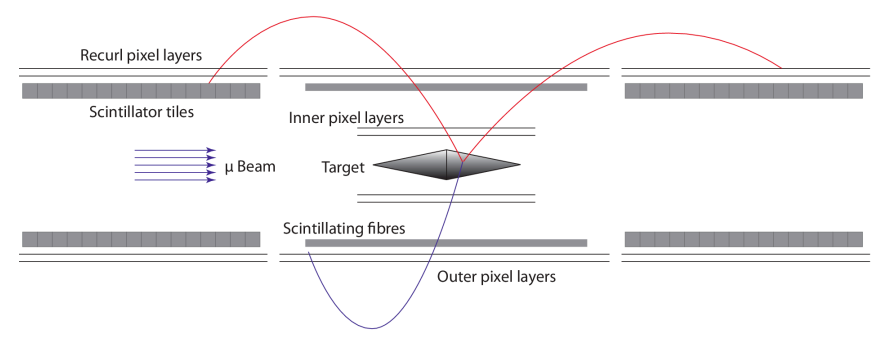

Figure 4: Schematic lognitudinal cross section of the proposed Mu3e detector.

\section{Silicon pixel detector technology}

For the precise measurements of both momentum and vertex position of low momentum electrons originating from muon decay, a tracking detector built from HV-MAPS will be implemented. The n-well acts as a charge collecting electrode and the charge collecting mechanism is mainly by drift in the depletion region of about $9 \mu \mathrm{m}$ thickness. This leads to relatively high MIP signals compared to standard MAPS, better timing and an improved radiation tolerance. The unique feature of the HV-MAPS is the placement of pixel electronics inside the collecting electrode as shown in figure 5. This allows 100\% fill factor. Both PMOS and NMOS transistors can be placed inside the charge collecting n-well using the twin-well option. Since the charge collection occurs in the 
thin depleted region, the HV-MAPS can theoretically be thinned up to nearly $30 \mu \mathrm{m}$ without significant signal loss. Furthermore, HV-MAPS can be implemented in a "cheap" commerical process.

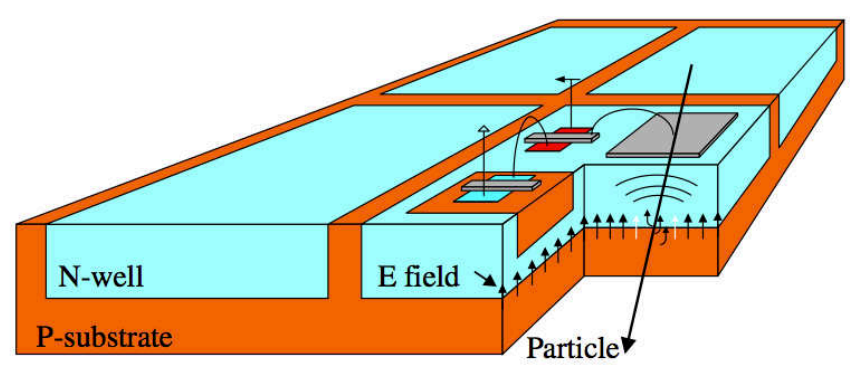

Figure 5: Schematic view of a HV-MAPS sensor [11]

AMS/IBM $180 \mathrm{~nm}$ HV-CMOS technology has been chosen here as it offers a very wide range of operating conditions and also a maximum reticle size of $2 \times 2 \mathrm{~cm}^{2}$.

\subsection{Design details}

HV-MAPS are based on the principle that analog and digital readout circuits are designed in a deep n-well which is implanted into the p-substrate and hence protected against the high voltage. The charge sensitive amplifier (CSA) is integrated into each pixel cell whereas the comparator and digital readout are placed at the chip periphery to minimize digital cross talk. A series of HV-MAPS prototypes, the MUPIX chips, have been produced for the Mu3e experiment and tested [12, 13, 14, The recently tested fourth generation of the MUPIX chip i.e the MUPIX 4 chip has $40 \times 32$ pixels, a pixel size of $80 \times 92 \mu \mathrm{m}^{2}$, and an active area of $9.4 \mathrm{~mm}^{2}$. It comprises a digital readout logic for zero-suppression. Every pixel is connected to its readout cell. The readout cell stores the hit bit and a time stamp if the pixel cell senses a particle hit. Inside each column a token is sent from one read out cell to the next. The pixel readout cell with an active hit bit sends its address and time stamp to the column memory while it has a token. Then the hit data of the MUPIX4 chip is read out via parallel data links by a FPGA. The next step in the MUPIX chip design will be to implement an embedded readout state machine and a fast serializer.

The mechanical support for the MUPIX chip has been optimized for low radiation length to minimize multiple scattering. Hence HV-MAPS are thinned down to $50 \mu \mathrm{m}$ and then glued and wire bonded to a $25 \mu \mathrm{m}$ thick flex-print with aluminum traces. This flex-print with the chips is mounted on a mechanical frame made of $25 \mu \mathrm{m}$ thick $\operatorname{Kapton}^{\mathrm{TM}}$ foil which is very light and sturdy [9].

\section{Performance of the MUPIX4 chip}

The MUPIX4 chip performance has been studied using test beam measurement at DESY in 2013-2014.

\subsection{Measurement setup}

The MUPIX4 chip has been tested using the EUDET telescope Aconite [15] at the DESY test beam line T22. The beam line provides electrons from converted bremsstrahlung photon beams 
which are generated by a carbon fibre target in the electron synchrotron DESY. The energy of the produced electons can be in the range of 1 to $6 \mathrm{GeV}$. This telescope is built from six layers of Mimosa26 MAPS thinned to $50 \mu \mathrm{m}$ each with spatial resolution of 2-3 $\mu \mathrm{m}$ [16]. The setup with the EUDET telescope and the MUPIX4 chip is identical to the one used in previous measurements [17]. Data were taken at electron momenta of $5 \mathrm{GeV} / \mathrm{c}$ to minimize multiple scattering effect in the track reconstruction. The device under test has been oriented with respect to the beam line at angles of $0^{\circ}, 22.5^{\circ}$ and $45^{\circ}$ resulting in a variation of the projected thickness $d_{\text {eff }}$ traverse by electrons is from 50 to $141 \mu \mathrm{m}$.

\subsection{Results from test beam measurements at DESY}

The time resolution of MUPIX4 was calculated by taking the time difference between the time-stamps of the chip and the tigger signal from the trigger logic unit (TLU). The time-stamp is measured with an external Gray counter with a frequency of $100 \mathrm{MHz}$. The measured time resolution is about $17 \mathrm{~ns}$, as shown in figure 6, which includes the time resolution of the DAQ. This time resolution fullfils the Mu3e experiment requirement which is a fine time counter frequency of 20 $\mathrm{MHz}$ [10].

The single hit resolution is calculated by projecting the target MUPIX4 chip perpendicular to the

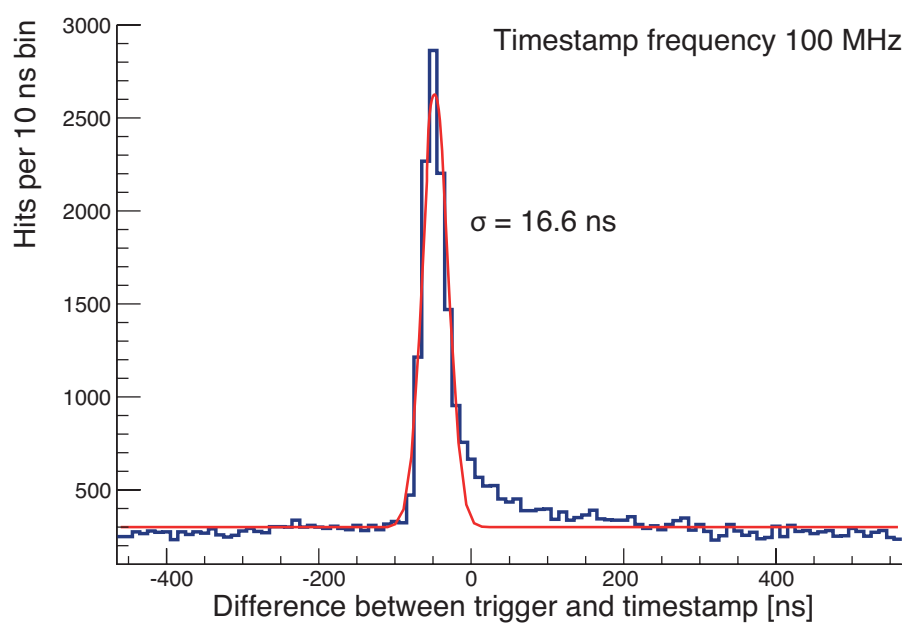

Figure 6: Time resolution of MUPIX4 chip and the red line is the Gaussian fit to the background.

beam line. The tracks are reconstruced separately in the upstream and downstream section of the telescope and interpolated to the the target plane. The track residual is measured as the difference between hit position and the expected hit position from the fitted track. The single hit resolution is determined by the pixel size and the measured track RMS residuals are $28 \mu \mathrm{m}$ and $29 \mu \mathrm{m}$, in the $\mathrm{x}$ and y direction respectively, as shown in figure 7. The contribution to track residual from multiple scattering is $19 \mu \mathrm{m}$.

The efficiency of the MUPIX4 chip is defined as the ratio of total number of tracks with an associated pixel hits to the total number of reconstructed tracks through each pixel. The efficiency of MUPIX4 chip is 99\% without tuning of single-pixel thresholds, as shown in figure 8 . Furthermore, the efficiency of the MUPIX4 chip has been measured by rotating the chip with re- 


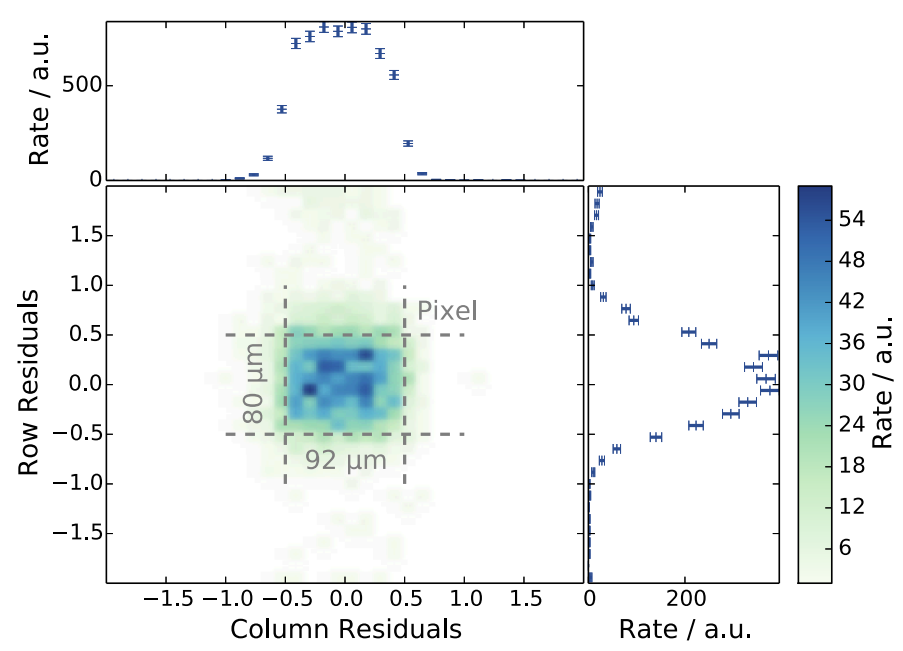

Figure 7: Single hit resolution in terms of column and row residuals for the MUPIX4 chip which is projected perpendicular to beam direction.

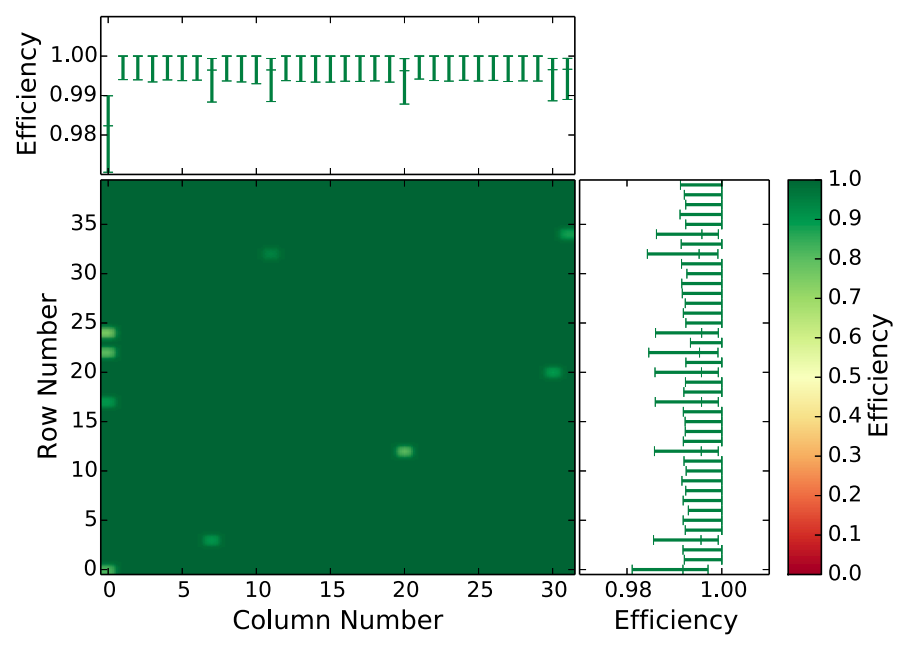

Figure 8: Efficiency of MUPIX4 chip for perpendicular tracks with a bias voltage of $70 \mathrm{~V}$ and a pixel threshold of 460 electrons.

spect to the beam line by $22.5^{\circ}$ and $45^{\circ}$. The efficiency is best for an angle of $45^{\circ}$ with respect to beam line since the line of sight thickness of the depletion zone is inversely proportional to the cosine of the angle of rotation of the chip with respect to the beam line. The efficiency results are summarized in figure g. Since the base line offset is $800 \mathrm{mV}$, a study of the efficiency of MUPIX4 chip has been perfomed as a function of readout threshold which is 20 electrons $/ \mathrm{mV}$.

\subsection{Readout feature}

The reset of a RAM cell in the pixel readout cell before the hit has been sent to the column memory causes a pixel address problem in one half column. Further investigations and simulation studies have been performed to understand this problem. The problem has been fixed in a new 


\section{Column matched efficiencies, $70 \mathrm{~V}$}

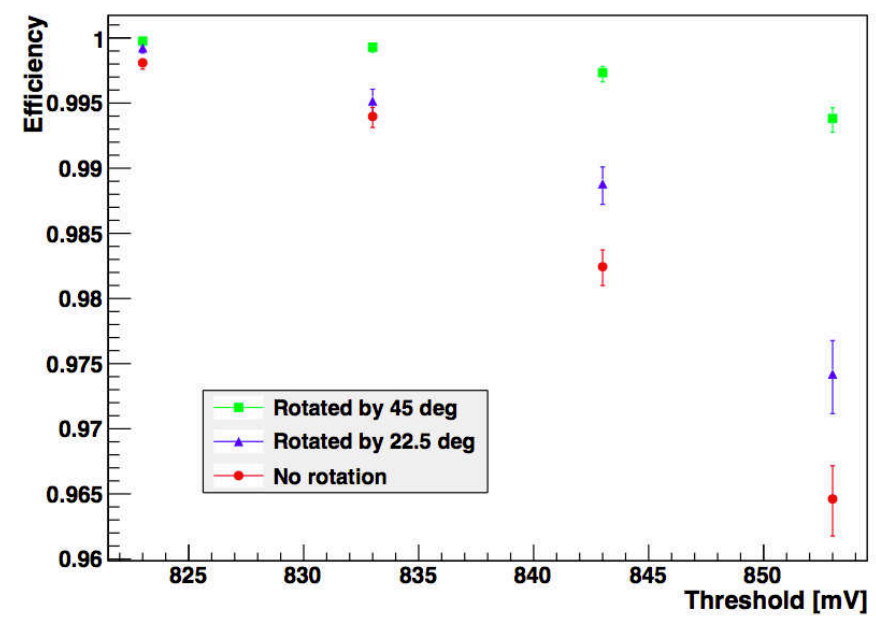

Figure 9: Efficiency of the MUPIX4 chip as a function of the readout threshold for different incidence angles.

version of the MUPIX chip, which is the MUPIX6 chip, using inverters as repeaters to adjust the time delay between the two signals which control the switch associated to the RAM cell.

\section{Summary}

The Mu3e experiment searches for the charged LFV process $\mu^{+} \rightarrow e^{+} e^{-} e^{+}$aiming for a sensitivity of $\mathrm{B}(\mu \rightarrow e e e)<10^{-16}$. A rate of $10^{9}$ muons per second and a very good background rejection are required to achieve this sensitivity. The choice of HV-MAPS sensor results in a tracking detector with a thickness of $\leq 0.1 \%$ of a radiation length. Also, fast charge collection efficiency and radiation hardness [18] can be achieved. The current version of the MUPIX prototype has demostrated the required analog performance. From test beam data collected with the MUPIX4 chip, the time resolution is $17 \mathrm{~ns}$, the single hit resolution is given by pixel size and is $80 \times 92 \mu \mathrm{m}^{2}$, the efficiency is above $99 \%$ for perpendicular tracks. The integration of full digital electronics into the MUPIX chip is expected to be completed by the end of 2014 and the production of larger sensor is expected by 2015 .

\section{References}

[1] Y. Fukuda et al, [Super-Kamiokande Collaboration], Evidence for oscillation of atmospheric neutrino, Phys. Rev. Lett. 81 (1998) 1562-1567.

[2] Q. R. Ahmad et al., [SNO Collaboration], Measurement of the Rate of $v_{e}+d \rightarrow p+p+e^{-}$ Interactions Produced by ${ }^{B} B$ Solar Neutrinos at the Sundbury Neutrino Observatory, Phys. Rev. Lett. 87 ( 2001 ) 071301.

[3] K. Eguchi et al., [KamLAND Collaboration], First Results from KamLAND: Evidence for Reactor Antineutrino Disappearance, Phys. Rev. Lett. 90 (2003) 021802. 
[4] Y. Kuno and Y. Okada, Muon Decay and Physics Beyond the Standard Model, Rev. Mod. Phys. 73 (2001) 151-202.

[5] J. Adam et al., New Constraint on the Existence of $\mu^{+} \rightarrow e^{+} \gamma$ decay, Phys. Rev. Lett. 110 (2013) 201801.

[6] W. H. Bertl et al. A search for $\mu$-e conversion in muonic gold, Eur. Phys. J. C47 (2006) 337-346.

[7] The CMS Collaboration, Search for lepton flavor violating decays of the Higgs boson, CMS Physics Analysis Summary CMS PAS HIG-14-005, 2014.

[8] U. Bellgardt et al., Search for the decay $\mu^{+} \rightarrow e^{+} e^{-} e^{+}$, Nucl. Phys. B299 1 (1988).

[9] N. Berger et at., A Tracker for the Mu3e Experiment based on High-Voltage Monolithic Active Pixel Sensors, Nucl. Instr. Meth. A732 (2013) 61-65.

[10] A. Blondel et al., Research Proposal for an Experiment to Search for the Decay $\mu \rightarrow$ eee (2013) (arXiv: :1301.6113 [physics.ins-det]).

[11] I. Peric, A novel monolithich pixelated particle detector implemented in high-voltage CMOS technology, Nucl. Instr. Meth. A582 (2007) 876-885.

[12] H. Augustin, Charakterisierung von HV-MAPS, Bachelor Thesis, University of Heidelberg, 2012.

[13] A. K. Perrevoort, Characterisation of High-Voltage Monolithic Active Pixel Sensors for the Mu3e Experiment, Master Thesis, University of Heidelberg, 2012.

[14] R. Philipp, Characterisation of High Voltage Monolithic Active Pixel Sensors for the Mu3e Experiment, Master Thesis, University of Heidelberg, 2014.

[15] P. Roloff, The EUDET high resolution pixel telescope, Nucl. Instr. Meth. A604 2009 265-268.

[16] J. Behr, Test Beam Measurement with the EUDET Pixel Telescope, Technical report, University of Hamburg, 2010.

[17] N. Berger et al., Multiple Columb Scattering in Thin Silicon, (2014) (arXiv: : 1405.2759 [physics.ins-det]).

[18] S. Gonzalez-Sevilla et al., Published in proceedings of Radiation-hard active pixel detectors based on HV-CMOS technology for HL-LHC upgrades, PoS (EPS-HEP 2013) 498. 